\title{
¿DÓNDE ESTÁY LOS HOMBRES? GÉNERO Y ESTRUCTURA OCUPACIONAL EN PUERTO RICO
}

\author{
Ángel L. Rivera Aponte \\ Víctor I. García Toro ${ }^{2}$ \\ Rafael L. Ramírez ${ }^{3}$
}

\section{Resumen}

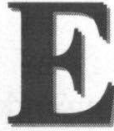

$\mathrm{n}$ este artículo se discute la participación masculina en la fuerza laboral de Puerto Rico para el año 2000 y la escolaridad de los hombres incorporados a la misma, por las respectivas ocupaciones y los grupos ocupacionales principales. Se realizó un análisis cruzado entre 650 ocupaciones y diez categorías de escolaridad. Además se identificaron aquellas ocupadas por los hombres que no tenían escolaridad. Entre los hallazgos sobresalen los siguientes: la fuerza laboral masculina estaba compuesta por 859,092 individuos, de los cuales 14,353 hombres en la fuerza laboral no poseían escolaridad; el 73.89 por ciento de dicha la fuerza laboral no poseía estudios post-secundarios; y que solamente 2.96 por ciento ostentaba el grado de primer nivel profesional o doctorado.

Descriptores: fuerza laboral masculina, nivel de escolaridad, planificación de recursos humanos y política pública.

\footnotetext{
${ }^{1}$ Catedrático Auxiliar en la Facultad de Administración de Empresas, Universidad de Puerto Rico en Río Piedras.

2 Catedrático en la Escuela Graduada de Trabajo Social Beatriz Lassalle, Universidad de Puerto Rico en Río Piedras.

${ }^{3}$ Catedrático Jubilado de la Universidad de Puerto Rico en Río Piedras (Q.D.P.)
} 


\begin{abstract}
The main purpose of this paper is to discuss male participation in the labor force in Puerto Rico for 2000 and the educational level of those men, according to occupations and principal occupational groups. A cruise analysis of 650 occupations and 10 educational levels was realized. The occupations held by men without education were also identified. The main findings of this paper are: the male labor force totaled 859,092 individuals of which 14,353 reported no education; $73.89 \%$ had no post-secondary education, and only $2.96 \%$ obtained degrees at the first professional level or doctorates.
\end{abstract}

Key words: male labor force, educational levels, planning of human resources and public policy.

\title{
Introducción
}

Los cambios notables en la estructura, composición y dirección de la fuerza laboral a nivel mundial en las ultimas décadas (Internacional Labor Force, 2004), apuntan a la necesidad de investigar los factores que inciden sobre la demanda del mercado laboral. Esas transformaciones requieren el desarrollo de políticas y programas que permitan a los gobiernos cumplir sus objetivos del modo más óptimo en cuanto a establecer un programa de planificación de los recursos humanos en el contexto de la fuerza laboral (Director, 1985). Esta política pública requiere de un amplio conocimiento de la oferta y demanda del mercado laboral a nivel micro y macro.

La planificación de los recursos humanos en el contexto de la fuerza laboral se desarrolló a principios de los años sesenta del pasado siglo (Hopkins 1988). A mediados de esa década, en Estados Unidos, se desarrolló una política federal sobre la fuerza laboral encaminada a la planificación de la misma. Esta se inició para tratar de detener la ola de críticas sobre organización y administración de programa y para responder al hecho de que los administradores federales no conocían las condiciones de los mercados locales. A finales de los setenta la planificación de la fuerza laboral comenzó a asumir un rol más amplio y se vinculó a la planificación estratégica y operacional pero, 
todavía se enfocaba en modelos de pronósticos que no tomaban en consideración factores tales como el ambiente político y social, condiciones organizacionales y posibles cambios en la dirección corporativa.

En la década de los sesenta Weisskoff y Wolf (1975) desarrollaron un proyecto en Puerto Rico, financiado por el Departamento del Trabajo Federal, encaminado a integrar la planificación de los recursos humanos con la planificación del desarrollo económico. Según señala Ruiz Mercado (1982, 1986, 1990) estos hicieron la primera integración del modelo de insumo-producto (usado como herramienta en la planificación del desarrollo económico) con las matrices ocupacionales (usadas en la planificación de los recursos humanos).

Un asunto que cobra cada vez más importancia en las investigaciones y proyecciones de la planificación de los recursos humanos en el contexto de la fuerza laboral es el género. Quiere decir, como se ubican los hombres y las mujeres en la estructura ocupacional y los cambios en ésta última generados por las transformaciones socioeconómicas. Las investigaciones acerca de la demanda y características del mercado laboral puertorriqueño se presentan de manera comparativa dentro de la categoría de género, prestando particular atención al género femenino. Las publicaciones que tratan el tema de la demanda del mercado laboral desde la perspectiva del género masculino son prácticamente inexistentes.

El propósito de este artículo es analizar la demanda del mercado laboral para el género masculino en Puerto Rico del 1960 al 2000. El análisis se presenta de manera agregada por los grupos ocupacionales principales y desagregados por el detalle de cada una de las ocupaciones. Entre los datos que presentamos aparecen las ocupaciones que desaparecieron durante ese período y aquellas en las cuales la participación masculina es menor de 50.0 por ciento así como los nuevos empleos por décadas para hombres que se generaron en esos 40 años. Tras la exposición de los hallazgos consideramos los factores que han provocado estos cambios dentro del mercado laboral para los hombres. 


\section{Metodología}

Las fuentes principales de datos fueron los documentos publicados por el Departamento de Comercio de los Estados Unidos de América, Negociado del Censo Federal; Censo de Población de Puerto Rico: para las décadas de 1960, 1970, 1980, 1990 y 2000. ${ }^{4}$ Información adicional solicitada a la Oficina de Censo Federal, Oficina del Censo de Puerto Rico, Junta de Planificación de Puerto Rico y la Universidad de Puerto Rico. De esas fuentes se recopilaron los datos concernientes a la clasificación ocupacional detallada del grupo trabajador civil diestro y de las personas empleadas por sexo.

Parte de los datos del Censo de 2000 se obtuvieron a través de llamadas telefónicas y comunicación electrónica con el Negociado del Censo Federal debido a que el banco de datos censales disponible en Puerto Rico estaba sujeto a ciertas restricciones. Estas últimas fueron específicamente en los datos informados de las ocupaciones e industrias. La información de clasificación industrial y ocupacional por género se recibió a mediados del mes de marzo del 2005 a través de una autorización del gobierno federal de los Estados Unidos, respondiendo a una petición especial de Rivera Aponte (2006) para utilizar los archivos de "Advance Query Systems". Estos archivos fueron trasladados al programa Excel de manera desagregada. Por su amplitud, se utilizó un sistema computadorizado especial para poder limpiarlos. La información fue clasificada en dos vertientes: ocupación por género y la ocupación por industria.

Se clasificaron todas las ocupaciones utilizando el Sistema de Clasificación Ocupacional de los Estados Unidos (1960, 1970, 1980, 1990 y 2000), usando diferentes niveles de agregación: grupo ocupacional principal, los subgrupos ocupacionales y el detalle de cada unas de las ocupaciones. La magnitud de las ocupaciones

\footnotetext{
${ }^{4}$ Los datos del Censo referente a los sectores ocupacionales no se encuentran en un solo lugar, los cual dificultó su localización y acceso. Los datos del Censo de 1960, 70 y 80 se encuentran en la Colección Puertorriqueña, Biblioteca Lázaro, UPR en Río Piedras. La información del Censo de 1990 está localizada en el Recinto de Ciencias Medicas de la Universidad de Puerto Rico y los datos del Censo de 2000, se encuentran en la Oficina del Censo en la Junta de Planificación de Puerto Rico.
} 
registradas en Puerto Rico a través de las décadas, los cambios en los nombres de las clasificaciones y de los códigos que establecen los estándares de clasificaciones ocupacionales (SOC), requirieron uniformar la base de datos.

Además se clasificaron cada una de las ocupaciones de manera detallada a través de las décadas. La cantidad de estas ocupaciones fluctuó entre 500 a 650 . Después de identificar los códigos y definiciones establecidas en el manual de clasificación ocupacional, se agregaron las ocupaciones dividiéndolas en diferentes categorías, lo que resultó en una agregación de 113 subgrupos ocupacionales. Estos subgrupos ocupacionales a su vez se reagruparon en 13 grupos ocupacionales principales: las ocupaciones ejecutivas, administrativas y gerenciales; las profesionales; las técnicas y de apoyo técnico; los vendedores; las de apoyo administrativo, oficinista, secretarias y otros; las de servicios; las de agricultura, silvicultura y pesca; reparadores y mecánicos; trabajadores diestros en la construcción; las ocupaciones en trabajos de precisión; los operarios de transportación y ocupaciones relacionadas con el movimiento de materiales y los trabajadores de la construcción y otros obreros. Una vez completada la recopilación y clasificación de los datos se hizo el análisis de los cambios en los empleos ocupados por el género masculino.

\section{Hallazgos}

De acuerdo con los datos del Censo de Puerto Rico para el año 2000 se informaron 1,569,934 personas empleadas, de las cuales 867,209 eran hombres, lo cual equivale a un 55.22 por ciento del total de la fuerza laboral. La tabla 1 presenta las 30 ocupaciones de mayor demanda en la cuales se ubicaron los hombres en el año 2000. Estas 30 ocupaciones representaban 53.61 por ciento de la fuerza laboral masculina que a su vez equivale a un 4.58 por ciento de un total de 655 ocupaciones registradas. 


\begin{tabular}{|c|c|c|}
\hline & Demanda & Por ciento \\
\hline Ocupaciones Género Masculino & 867,209 & $53.61 \%$ \\
\hline Conserjes y Empleados de Limpieza & 36,086 & $4.16 \%$ \\
\hline Conductores de Camiones, Pesados y Livianos & 32,153 & $3.71 \%$ \\
\hline Obreros de Construcción & 31,746 & $3.66 \%$ \\
\hline Guardias y Policía, exceptuando Servicio Público & 24,987 & $2.88 \%$ \\
\hline Trabajadores en venta, Otros Productos & 21,879 & $2.52 \%$ \\
\hline Carpinteros & 20,843 & $2.40 \%$ \\
\hline Mecánicos de Automóvil & 20,120 & $2.32 \%$ \\
\hline Otros Trabajadores de Producción, incluyendo procesadores de de semiconductores y equipo de refrigeración & 19,423 & $2.24 \%$ \\
\hline Supervisores y Propietarios, Ocupaciones de Ventas, Asalariados & 19,005 & $2.19 \%$ \\
\hline Manipuladores de Provisiones y Ensacadores & 17,544 & $2.02 \%$ \\
\hline Trabajadores Agrícolas & 16,087 & $1.86 \%$ \\
\hline Representantes de Ventas de Minería, Manufactura y Comercio al por Mayor & 16,007 & $1.85 \%$ \\
\hline Albañiles, Mamposteros & 13,838 & $1.60 \%$ \\
\hline Cajeros & 13,573 & $1.57 \%$ \\
\hline Ayudantes, Rama de Construcción & 13,002 & $1.50 \%$ \\
\hline Policía y Detectives, Servicio Público & 12,337 & $1.42 \%$ \\
\hline Contables y Auditores & 11,952 & $1.38 \%$ \\
\hline Cocineros & 11,924 & $1.37 \%$ \\
\hline Supervisores, Ocupaciones de Producción & 11,485 & $1.32 \%$ \\
\hline Encargados de Terrenos y Jardineros, excluyendo fincas & 11,450 & $1.32 \%$ \\
\hline Supervisores, Oficina en General & 10,777 & $1.24 \%$ \\
\hline Oficinista de Almacenace y Llenadores de Ordenes & 10,284 & $1.19 \%$ \\
\hline Adm. y Ger., Asalariados N.C.O.C. & 10,047 & $1.16 \%$ \\
\hline Supervisores y Propietarios, Ocupaciones de Ventas, Auto-Empleados & 9,548 & $1.10 \%$ \\
\hline Operadores de Equipo Miscelaneo de Contruccion & 8,581 & $0.99 \%$ \\
\hline Oficinistas Generales & 8,461 & $0.98 \%$ \\
\hline Maestros, Escuela Elemental & 8,460 & $0.98 \%$ \\
\hline Conductores de Taxi y Choferes & 8,398 & $0.97 \%$ \\
\hline Supervisores y Administradores de Ocupaciones de Construcción y Trabajadores de Extracción & 8,224 & $0.95 \%$ \\
\hline Reparadores de Maquinaria Industrial & 6,691 & $0.77 \%$ \\
\hline
\end{tabular}

La distribución agregada de los grupos ocupacionales principales para el género masculino en el año 2000 aparece en la tabla 2. La mayoría de los hombres estaban ubicados en los siguientes grupos ocupacionales principales: servicios; trabajadores en servicios de limpieza y otros obreros trabajadores de la construcción; vendedores; y trabajadores diestros de la construcción. Estos cuatro grupos representaban un 47.02 por ciento del total de los empleos ocupados por los hombres. El total de la demanda para las ocupaciones que, a nuestro juicio, requieren mayor preparación académica fueron las categorías de la I a la IV y comprendieron el 28.70 por ciento de la demanda ocupacional. 


\begin{tabular}{|c|c|c|}
\hline \multicolumn{3}{|l|}{$\begin{array}{l}\text { Tabla 2: Demanda Ocupacional Agregada para el Género Masculino } \\
\text { Puerto Rico, Censo } 2000\end{array}$} \\
\hline \multirow[b]{2}{*}{ Grupo Ocupacional } & \multicolumn{2}{|c|}{ Masculino } \\
\hline & 2000 & Por Ciento \\
\hline Demanda Total & 867,209 & $100.00 \%$ \\
\hline I. Ocupaciones Ejecutivas, Administrativas y Gerenciales & 65,706 & $7.58 \%$ \\
\hline II. Profesionales & 70,311 & $8.11 \%$ \\
\hline III. Ocupaciones Técnicas y de Apoyo Técnico & 15,986 & $1.84 \%$ \\
\hline IV. Vendedores & 96,899 & $11.17 \%$ \\
\hline V. Ocupaciones de Apoyo Administrativo & & \\
\hline Oficinistas, Secretarias y Otros & 75,190 & $8.67 \%$ \\
\hline VI. Ocupaciones de Servicios & 130,862 & $15.09 \%$ \\
\hline VII. Ocupaciones en Agricultura, Silvicultura y Pesca & 36,661 & $4.23 \%$ \\
\hline VIII. Reparadores y Mecánicos & 59,304 & $6.84 \%$ \\
\hline DX. Trabajadores Diestros en la Construcción & 77,001 & $8.88 \%$ \\
\hline X. Ocupaciones en Trabajos de Precisión & 29,744 & $3.43 \%$ \\
\hline XI. Operarios de Máquina, Armadores e Inspectores & 41,644 & $4.80 \%$ \\
\hline XII. Operarios de Transportación y Ocupaciones Relacionadas & & $0.00 \%$ \\
\hline con el Movimiento de Materiales & 63,247 & $7.29 \%$ \\
\hline XIII. Trabajadores en Servicio de Limpieza y Otros Obreros de la Construcción & 102,992 & $11.88 \%$ \\
\hline A. Otros obreros categorias diversas_1/ & 1,662 & $0.19 \%$ \\
\hline B. Desempleados, sin Reciente Experiencia de Trabajo Civil & 49,582 & $5.72 \%$ \\
\hline
\end{tabular}

Las transformaciones en la estructura ocupacional del género masculino durante el periodo de 1960 al 2000 apuntan a la desaparición de ocupaciones. De acuerdo al Censo de 1960 no se registró participación en las siguientes ocupaciones: fogoneros, cadeneros, portamiras, preparadores de estacas, instaladores de equipos de barcos, listoneros, operarios de tren, varios renglones de trabajadores de almacén, entejadores, conductores de ferrocarril, guarda almacén en fincas, herraderos, peleteros, forjadores, martilleros y mosaiqueros.

En el Censo de 1970 no aparecen las siguientes ocupaciones: bruñidores, cuneteros, embotelladores y enlatadores, escogedores y clasificadores de manufactura, trabajadores de muelles y estibadores, carreteros, cocheros, cuarteros, limpiabotas, monitores escolares, oficiales de logia y sociedades, boteros y lancheros, trabajadores en asbestos e instaladores de aisladores, aceiteros y engrasadores, excepto en autos. 
En el Censo de 1980 no se informaron ocupaciones desaparecidas y en el de 1990 se identificaron solamente como desaparecidas las siguientes: maestros de economía doméstica, aprendiz de plomero, instalador de tubería y máquina de vapor, taladradores de pozos de petróleo. Sin embargo es interesante referirse a Standing (1989), sobre la participación de los hombres en la fuerza laboral que afirmaba que la misma se había reducido o estancado y tenía como una de sus razones principales la mayor participación de la mujer en la fuerza laboral. Para el año 2000 desaparecieron las siguientes ocupaciones: técnicos de ingeniería mecánica; programadores de instrumentos de control numérico; operadores de equipo periférico; taquígrafos; operadores de frenos, señales y cambios de vía. Entre los años 1960 a 2000 desaparecieron 59 ocupaciones.

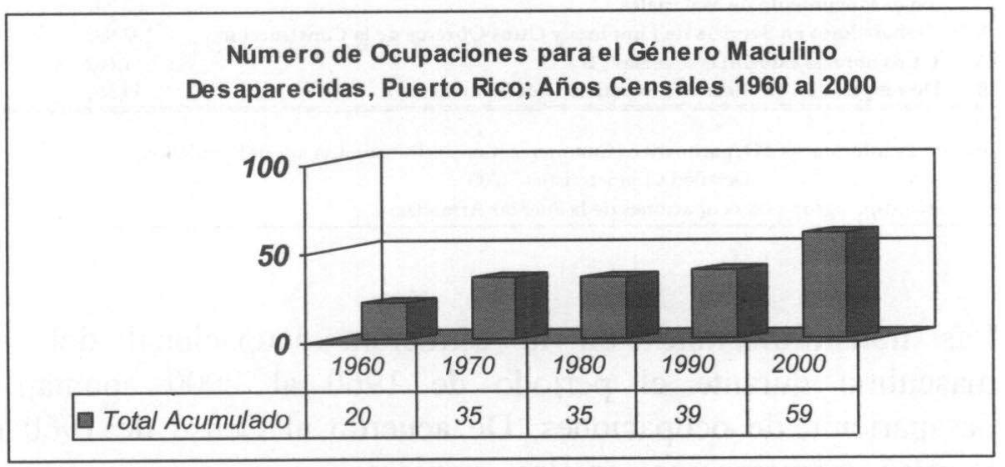

En las últimas cuatro décadas del siglo $\mathrm{XX}$ encontramos una progresiva disminución de la participación masculina en las ocupaciones registradas en Puerto Rico. Safa (1998) comenta que esta disminución fue acompañada por "una mayor participación de las mujeres debido a la industrialización que elevó la participación de la mano de obra femenina, por el aumento de su educación, la disminución de la fecundidad y la ampliación de la oferta de mano de obra femenina cualificada" (p.47).

En el 1960 aparecen 28 ocupaciones entre las cuales se encuentran: administradores de servicios Educativos, administradores médicos y de salud, enfermeras registradas, dietistas, asistentes de médicos, 
maestros de diferentes niveles, trabajadores sociales, consejeros vocacionales, especialistas en servicios comunitarios, editores y reporteros, bibliotecarios, técnicos de laboratorio y radiología, cajeros, secretarios, taquígrafos, mecanógrafos, recepcionistas, entrevistadores, oficinistas, lavanderos, planchadores, amas de llave y mayordomos, limpiadores de Hogares, cocineros, peluqueros y cosmetólogos, trabajadores en cuidado de niños, costureras, inspectores de producción. En 1970 se encontraron cinco nuevas ocupaciones: sociólogos, planificadores urbanos, maestros de inglés, oficinistas estadísticos, oficinistas de bibliotecas.

En 1980 se identificaron 51 ocupaciones adicionales (Vea tabla 1 en Apéndice 1); en el 1990 se añadieron 27 ocupaciones (Vea tabla 2 en Apéndice 1). En el 2000 se identificaron otras 71 ocupaciones (Vea tabla 3 en Apéndice 1).

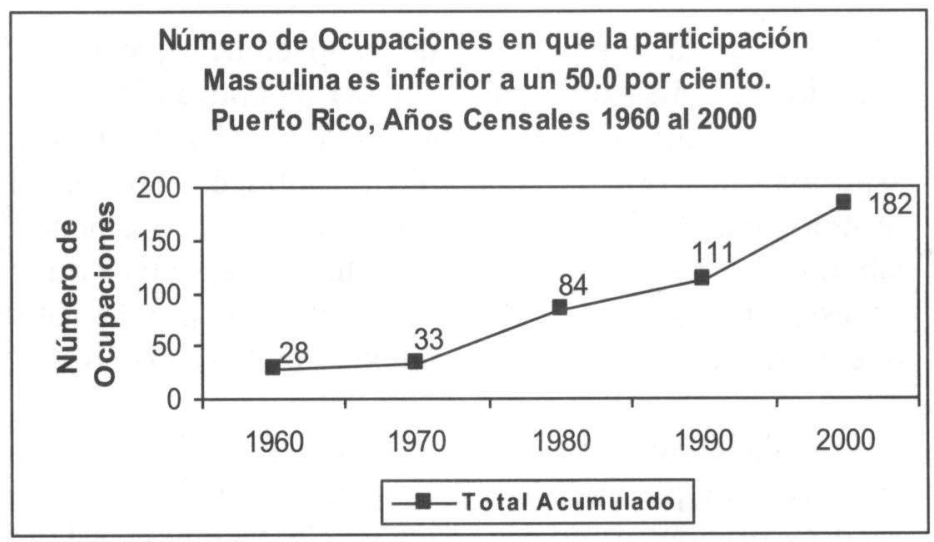

En los informes de los Censos en Puerto Rico que analizamos, se registraron un sinnúmero de categorías ocupacionales en las cuales copaba el género masculino con un 100 por ciento de participación (Vea ocupaciones para los años 1960 en tabla 1, Apéndice 2). En el 1970, sólo seis nuevas ocupaciones tenían 100 por ciento de participación masculina: Maestros de Física; Maestros de Ciencias Políticas; Técnicos de Ciencia; Programadores de Instrumentos, Control Numérico; Reparadores de Equipo de Procesamiento de Datos. En el 1980 aumentaron estas ocupaciones controladas por los 
hombres (Vea ocupaciones en tabla 2 - Apéndice 2). En el 1990 fueron: Correspondencia y Manejo de Papeles; Constructores de Patrones y Modelos, Madera. En el 2000 era: Marineros en Buques Petroleros.

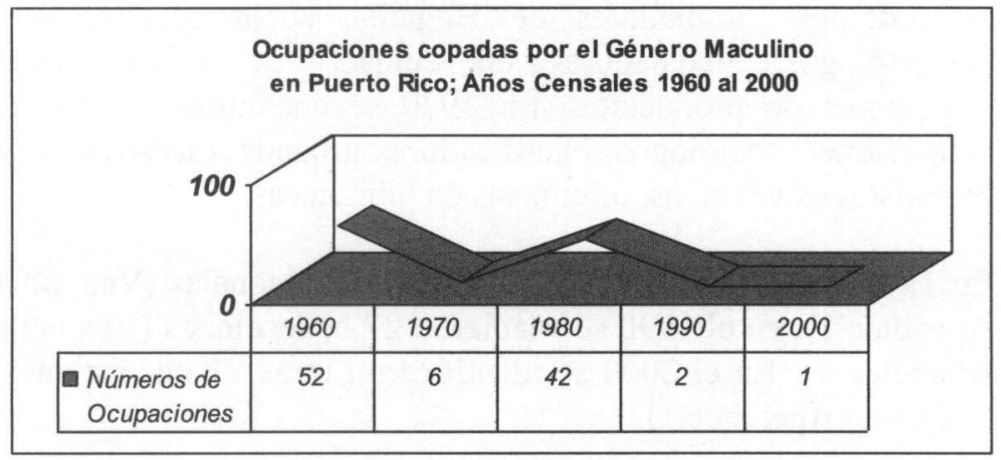

Más allá de la desaparición de ocupaciones que han impactado la participación masculina en la fuerza laboral en Puerto Rico durante las últimas cuarto décadas 1960 al 2000, se hace evidente el efecto de aspectos de orden económico, político, social, demográfico, tecnológico, geográfico, entre otros, sobre el fenómeno del mercado laboral. Ciertamente han sido muchas las variables que han incidido en este proceso que lo han hecho diverso por la naturaleza de la interrelación entre estas y el contexto donde se insiere.

Sin pretender ser exhaustivo en este análisis debemos reconocer que el proceso de planificación de los recursos humanos y sus efectos en la desaparición o no de nuevas ocupaciones, esta directamente vinculado a los procesos de planificación gubernamental y desarrollo de políticas públicas e ineludiblemente a los modelos económicos que promueven, rigen y a su vez guían el desarrollo económico de un país. En el caso de Puerto Rico los modelos de desarrollo económico que nos llevaron desde Manos a la Obra, Industria Liviana, Industria Pesada y Alta Tecnología en el momento actual, han generado en su implementación cambio a través de las políticas públicas que los propiciaron. Se suma a lo antes expuesto, los impactos de estas en el surgimiento y desaparición de nuevas industrias y ocupaciones que han sido generadas y sus efectos sobre el campo ocupacional y a su 
vez sobre la variable género en su sentido amplio, entre otros aspectos de interés.

Estos procesos de transformación de bases predominantemente económica, traen consigo a su vez cambios que inciden sobre lo político, lo social y los discursos preponderantes en la sociedad. En lo referente a la variable social, los cambios resultaron ser decididamente más evidentes, resaltando entre otros el incremento de la participación femenina en los mercados laborales de país desde la década de 1960 al presente. Este efecto aparentemente simple ha generado otra serie de cambios en cadena que han repercutido de modo complejo en la sociedad puertorriqueña. Pues si bien es cierto que estos cambios implicaron en una mayor participación femenina en los mercados de empleos y una mayor oportunidad de equidad al hacer accesible a la mujer a nuevos campos de acción no considerados antes, también trajeron consigo una serie de problemas sociales preocupantes.

Respecto al impacto de las políticas públicas que se vincularon al fenómeno antes mencionado, no se considero el efecto de este proceso de apertura de opciones al género femenino en el campo laboral sobre una sociedad estructuralmente poco preparada para enfrentarlos. Nos referimos al impacto de la participación de mujer a los nuevos campos laborales sin tener la infraestructura necesaria para lidiar con cambios como los siguientes: la estructura familiar, la importancia de la figura femenina en el sustento del hogar y la crianza de los hijos, el cambio en los roles femeninos y masculinos vinculados a la provisión de recursos y la nueva imagen de una mujer que era a sus vez madre y trabajadora. Es importante destacar que el efecto de este proceso sobre la estructura familiar fue y ha sido cuestionable y de modo general han tendido a recaer sobre la figura de la mujer los efectos adversos de este proceso de planificación.

De la misma forma, debemos ponderar que no se preparó al hombre que hasta el momento se consideraba el principal proveedor de hogar para lidiar con estos cambios y menos para verse paulatinamente desplazado de muchos de los sectores que habían estado antes bajo su dominio. Debe ser considerado que hasta el momento no se ha 
estudiando este fenómeno de desplazamiento y participación de los hombres en el mercado laboral desde la perspectiva de género como ha sido el caso de la mujeres y menos desde la perspectiva de la planificación de los recursos humanos entre géneros. Ciertamente este análisis del impacto que ha tenido sobre la estructura ocupacional de los hombres en Puerto Rico durante las últimas cuatro décadas estaría incompleto sin considerar su contraparte femenina. A pesar de que el propósito del artículo no es la comparación, huelga hacer una serie de consideraciones fuera del contexto de la planificación de los recursos humanos que era su meta. A tales efectos las aportaciones de Safa (1998) respecto a la mujer obrera durante las décadas del 1940 al 1990 nos evidencia una serie de aspectos relevantes que merecen ser considerados. Inicialmente a la autora le interesaba desmitificar la figura del hombre como proveedor y los datos que aportan en su investigación correspondientes al Caribe (Cuba, República Dominicana y Puerto Rico) inciden sobre los datos del análisis de este artículo al corroborar que la figura femenina ha estado paulatinamente aumentando su participación laboral en cuanto la del hombre ha ido disminuyendo sin que necesariamente esto haya implicado en una total equidad en término de género y acceso al mundo laboral (Safa, 1998).

Llama la atención los datos presentados por Safa (1998), sobre el aumento de la fuerza laboral femenina de un 18.0 a un 26.0 por ciento del 1950 al 1980, el cual fue superior al de los hombres en todos los grupos de edad en ese periodo. Entre otras causas, Safa (1998) trae a colación las siguientes:

.....el aumento en la educación femenina, el descenso en la fertilidad, la mayor migración rural y urbana y el aumento en la demanda de mano de obra femenina. A estas suma también aspectos como: la competencia internacional, las desregulación del trabajo, las emergentes políticas de ajustes estructurales que debilitan el poder de negociación del trabajador y de la industrialización por invitación (págs. 3-9). 
Concordamos con esos aspectos, los cuales consiguen promover cambios que afectan, de modo general, no solo a las mujeres sino también a los hombres.

Safa (1998) concluye planteando una serie de factores que son neurálgicos para explicar el cambio que se ha observado en la compasión por género de la fuerza laboral en Puerto Rico: "como primer factor hace referencia inicial a las estrategias de desarrollo basadas en la industrialización impulsadas por exportaciones y el aumento del sector de servicio que favorecen el empleo femenino sobre el masculino" (p. 227). Al respecto los trabajos de Rivera Aponte, Ruiz Mercado y García Toro (2007) comprueban sus efectos sobre la fuerza laboral de modo general y de los hombres de modo particular. Como segundo factor, Safa (1998) nos trae a consideración: "el aumento en la disponibilidad de mano de obra femenina gracias a la elevación de los niveles educativos, la reducción de la fecundidad y los rápidos procesos de industrialización" (p. 228). Ciertamente estos aspectos han incidido de modo claro y contundente en la composición de la fuerza laboral. Trabajo como los de Rivera Aponte (2006), Rivera Aponte, García Toro y Ramírez (2006), Rivera Aponte, Ruiz Mercado y García Toro (2007) y Rivera Aponte y López de Méndez (2009 en proceso de publicación), concuerdan con lo expuesto anteriormente.

De la misma forma, concurrimos con Safa (1998), cuando se refiere al tercer factor: "la crisis económico obligo a la mujeres a ingresar el mercado laboral como forma de hacer frente a los aumentos del costo de vida y la decreciente capacidad de los hombre como proveedores" (p. 229). Este factor esta claramente contemplado en nuestro análisis al evidenciar la creciente merma en la participación de los hombres en el mercado laboral, sea por desplazamiento o por ajustes a las nuevas políticas y exigencias de los mercados emergente a niveles nacional e internacional.

Se hace referencia también al impacto negativo de estrategias de desarrollo económico impulsadas por el estado (Ruiz Mercado, 1980; 1982), sobre el empleo masculino que han sido particularmente demoledora en el caso de Puerto Rico (Safa, 1998). Este impacto 
negativo ha sido evidenciado en este trabajo, particularmente en lo referente a la desaparición de miles de empleos tradicionalmente masculinos en las últimas cuatro décadas. Ciertamente ha quedado demostrado el impacto que tiene el desarrollo de políticas económicas sobre el proceso de re-estructuración económica, la planificación de los recursos humanos y su ineludible y estrecho vínculo sobre la dimensión social y especialmente sobre los géneros y sus diferencias ya marcada por los discursos que las encarnan.

\section{Conclusiones}

Los cambios en la participación masculina en la fuerza laboral durante los últimos cuarenta años del Siglo $\mathrm{XX}$ responden a varios factores, entre los cuales se destacan las transformaciones económicas generadas por las políticas gubernamentales para promover la industrialización y el empleo y las estrategias de inversión impulsadas por el capital desarrollista. Esos cambios en los sectores ocupacionales e industriales generaron nuevas ocupaciones, modificaciones y desaparición de otras respondiendo a los procesos antes mencionados y a la exigencia de nuevas destrezas y competencias dentro del mercado laboral. Los datos que analizamos en este artículo demuestran que en esas décadas la participación masculina en la fuerza laboral evidenció progresivas modificaciones, que culminaron en el año 2000 con: la desaparición de ocupaciones tradicionalmente masculinas, reducción de la participación de los hombres en el mercado laboral y una alta ubicación en ocupaciones no diestras para las cuales se requiere poca o ninguna educación post secundaria. Esto implica que en la fuerza laboral femenina se ha promovido un crecimiento acelerado de participación en sectores industriales y ocupaciones tradicionalmente masculinos, sin que esto signifique que al día de hoy todavía existan diferencias en equidad salarial, toma de decisiones y en ocupaciones de alta gerencia, entiéndase ejecutivas, administrativas y gerenciales (Rivera-Aponte, Ruiz Mercado y García Toro, 2007).

Las transformaciones de la fuerza laboral masculina en el período estudiado responden también a una concatenación de fuerzas sociales. Además de las exigencias del mercado de empleo en términos de 
destrezas y competencias, hay que considerar factores sociales y culturales entre los cuales se destacan: la incursión de las mujeres en ese mercado, reducción de la participación del género masculino en la educación post secundaria, la marginación del mundo del trabajo, los posibles efectos de la emigración y la intervención del Estado asistencialista, entre otros. Resta preguntar cual ha sido la mediación de factores culturales en los cambios en los significados de ser hombre, en su función como proveedor y el valor del trabajo en la construcción de las identidades masculinas. 


\section{Referencias}

Departamento de Comercio de los Estados Unidos de América, Negociado del Censo Federal. (1960). Censo de Población de Puerto Rico. San Juan Puerto Rico: Junta de Planificación, Programa de Planificación Económica y Social, Oficina del Censo.

Departamento de Comercio de los Estados Unidos de América, Negociado del Censo Federal. (1970). Censo de Población de Puerto Rico. San Juan Puerto Rico: Junta de Planificación, Programa de Planificación Económica y Social, Oficina del Censo.

Departamento de Comercio de los Estados Unidos de América, Negociado del Censo Federal. (1980). Censo de Población de Puerto Rico. San Juan Puerto Rico: Junta de Planificación, Programa de Planificación Económica y Social, Oficina del Censo.

Departamento de Comercio de los Estados Unidos de América, Negociado del Censo Federal. (1990). Censo de Población de Puerto Rico. San Juan Puerto Rico: Junta de Planificación, Programa de Planificación Económica y Social, Oficina del Censo.

Departamento de Comercio de los Estados Unidos de América, Negociado del Censo Federal. (2000). Censo de Población de Puerto Rico. San Juan Puerto Rico: Junta de Planificación, Programa de Planificación Económica y Social, Oficina del Censo.

Director, Steven M. (1985). Strategic Planning for Human Resource. Work in American Institute Studies in Productivity. New York. Pergamon Press. 
Hopkins, Michael, J. (1988). Employment Forecasting: The Employment Problem in Industrialized Countries. London, International Labor Organization.

Rivera Aponte, Ángel L. y López de Méndez, Annettee. (2008). Escolaridad y Ocupación de la Fuerza Laboral Femenina en Puerto Rico. Cuaderno de Investigación en la Educación, 23. Río Piedras: Centro de Investigaciones Educativas, Facultad de Educación, Universidad de Puerto Rico.

Rivera Aponte, Ángel L., Ruiz Mercado, Ángel L. y García Toro, Víctor I. (2007). Mujer y Brecha Salarial: Reto del Siglo XXI. San Juan. Puerto Rico: Oficina de la Procuradora de la Mujeres.

Rivera Aponte, Ángel L., García Toro, Víctor I. y Ramírez, Rafael L. (2006). Escolaridad y Ocupaciones en la Fuerza Laboral Masculina en Puerto Rico. Cuaderno de Investigación en la Educación, 21. Río Piedras: Centro de Investigaciones Educativas, Facultad de Educación, Universidad de Puerto Rico.

Rivera Aponte, Ángel L. (2006). Cambios en la Estructura Industrial y Ocupacional del Empleo en el Proceso de Desarrollo Económico de Puerto Rico: un Análisis Histórico de la Planificación de los Recursos Humanos usando el Modelo de Insumo-Producto del 1960 al 2000. Disertación Doctoral no publicada, Departamento de Ciencias Económicas y Administrativas, Universidad Interamericana de Puerto Rico, Recinto Metro.

Ruíz Mercado, Ángel L. (1980). Transformación Estructural en el Sector de los Servicios de la Economía de Puerto Rico. Cambios en el patrón de empleo femenino pro el sector industrial y por ocupación durante el proceso de desarrollo económico en Puerto Rico. Centro de Investigación Económica. Ensayos y Monografias, Revista de Ciencias Sociales, 18, Junio. 
Ruíz Mercado, Ángel L. (1982). Transformación Estructural en el Sector de los Servicios de la Economía de Puerto Rico. Ensayos y Monografias, Revista de Ciencias Sociales, 21 (2), marzo.

Ruíz Mercado, Ángel L. (1986). Requerimiento de Recursos Humanos por Ocupación y Sector Industrial Años 1984-1990. Puerto Rico: Occupational Information Coordinating Comite (P.R.O.I.C.C). San Juan: E.L.A.

Ruíz Mercado, Ángel L. (1990). Aplicaciones del modelo de insumo producto a la economía de Puerto Rico. Ensayos y Monografias. Revista de Ciencias Sociales, 55, marzo.

Safa, Helen I. (1998). De Mantenidas a Proveedoras: Mujeres e Industrialización en el Caribe. San Juan. Editorial de la Universidad de Puerto Rico.

Standing, Guy. (1989). Global Feminization through Flexible Labor. World Development, 17 (7): 1077-1096.

U.S. Department of Commerce Office of Federal Statistical Policy and Standards. (1960). Standard Occupational Classification Manual. Washington, D.C.: U.S. Government Printing Office.

U.S. Department of Commerce Office of Federal Statistical Policy and Standards. (1970). Standard Occupational Classification Manual. Washington, D.C.: U.S. Government Printing Office.

U.S. Department of Commerce Office of Federal Statistical Policy and Standards. (1980). Standard Occupational Classification Manual. Washington, D.C.: U.S. Government Printing Office.

U.S. Department of Commerce Office of Federal Statistical Policy and Standards. (1990). Standard Occupational Classification Manual. Washington, D.C.: U.S. Government Printing Office. 
U.S. Department of Commerce Office of Federal Statistical Policy and Standards. (2000). Standard Occupational Classification Manual. Washington, D.C.: U.S. Government Printing Office.

Weisskoff, Richard y Wolf, Edgard. (1975, November). Development and Trade Dependence: The Case of Puerto Rico, 1948-1963. The Review of Economic and Statistic. Boston: University of Harvard. 


\section{Apéndice 1}

\section{Tabla 1 - Ocupaciones aparecidas en 1980}

Científicos Matemáticos, Científicos Biológicos y de Vida, Optómetras, Otros Practicantes en Diagnóstico de Salud, Farmacéuticos, Terapeutas Respiratorios, Terapeutas Ocupacionales, Terapeutas Físicos, Terapeutas del Habla, Maestros: Economía, Ciencias Sociales, Ciencias, Computadoras, Especialistas en Salud, Idiomas Extranjeros, Archivistas y Curadores, Enfermeros Prácticos con Licencia, Higienistas Dentales, Técnicos y Tecnólogos de Registro de Salud, Asistentes Dentales, Trabajadores en venta, Otros Productos al Detal, Demostradores, Promotores y Modelos, Otras Ocupaciones de Apoyo en Ventas, Telemercadeo, Agentes de Reservaciones y Emisión de Billetes de Transportación, Oficinistas de Información en áreas relacionadas, Tenedores de Libros y Oficinistas de de Contabilidad y Auditoria, Oficinistas de Cobro, Operadores de Máquinas de Cobros, Posteros y Calculadoras, Suministradores de
Datos a la Computadora, Oficinistas de Elegibilidad, Seguro Social, Oficinista de Personal, excepto nómina y Marcadores de Tiempo, Oficinistas de Registro (Expedientes), Supervisores, Preparación de Alimentos y Ocupaciones de Servicio, Ayudantes de Mozos (as), Supervisores, Ocupaciones de Servicio de Personal, Ayudantes de Servicio de Transportación Pública, Fabricantes de Patrones, Trabajadores de Trazado y Cortadores, Encuadernador de Libros, Ensambladores de Equipo Eléctrico y Electrónico y Plástico, Misceláneos, Operadores de Máquinas de Coser Textiles, Operadores de Máquinas de Zapatos, Operadores de Máquinas Procesadoras, Ensambladores, Ensambladores Eléctricos, Electrónicos y Electromecánicos, Pasadores y recogedores de Muestras de Producción, Graduadores y Sorteadores, excepto Agrícolas 


\section{Tabla 2 - Ocupaciones aparecidas en 1990}

Químicos; excluyendo Bioquímicos, Economistas, Psicólogos, Autores, Escritores Técnicos, Maestros: Ciencias Biológicas, Ciencias Naturales, Historia, Ciencias Matemáticas, Arte, Drama y Música, Maestros Post-Secundaria, Otros Técnicos y Tecnólogos de Salud, Oficinistas de Nómina y del Reloj Registrador, Oficinistas de Costos y Tarifas, Expedidores, Ocupaciones de Apoyo Administrativo, Pagadores en Bancos, Operadores de Equipo
Comercial, N.C.O.C., Ayudantes de Servicio de Bienestar, Trabajadores en Ropa y Textiles, Misceláneos, Operadores de Máquinas Embaladoras y de Empacar, Operadores de Máquinas de Doblar, Operadores de Máquinas Rebanar y Cortar, Ayudantes, Ocupaciones Extractivas, Alimentadores de Máquinas y Portadores, Obreros, excluyendo de Construcción, Manufactura Bienes No-Duraderos

Tabla 3 - Ocupaciones aparecidas en el 2000

Administradores Financieros, Gerentes de Organizaciones de Servicios, Administradores de Propiedad, Bienes Raíces y Asociaciones Comunitarias, Administradores de Servicios Sociales y Comunitarios, Administradores y Agentes de Artistas, Entretenimientos y Atletas, Administradores de Base de Datos, Contables y Auditores, Especialistas en Personal, Adiestramiento y Relaciones Laborales, Inspectores de Seguros, Ocupaciones relacionadas con la Administración, Analistas de Créditos, Analistas Financieros, Examinadores Financieros, Oficiales
Supervisores de Oficinistas de Distribución, Scheduling y Ajuste, Supervisores y Administradores de Trabajadores de Apuestas, Entrevistadores, excepto elegibilidad y Préstamo, Entrevistadores Oficinistas de Prestamos, Oficinistas Hoteleros, Ajustadores de Seguro, Examinadores e Investigadores, Correctores de Pruebas, Consejeros Residenciales, Oficinistas de Manejos, Agentes de Viajes, Trabajadores de Apuestas, Oficinistas de Nuevas Cuentas, Oficinista de Corte Municipal y Licencias, Representantes de Servicios al Clientes, Autorizadores, 
Tabla 3 - Ocupaciones aparecidas en el 2000

y Consejeros de Créditos, Ingenieros Petroleros, Investigadores y

Analistas de Operaciones y

Sistemas, Estadísticos, Científicos

Físicos, Audiólogos, Diseñadores,

Bailarines, Trabajadores

Misceláneos de Medios y

Comunicaciones, Técnicos de

Laboratorios Dentales y Enseres

Médicos, Ayudantes Legales, Venta de Seguros, Bienes Raíces, Venta de Valores y Servicios Financieros, Publicidad y Otras Ocupaciones de Venta Relacionadas, Trabajadores en Venta de Ropa, Dependiente de Ventas, Supervisores, Operadores de Equipo de Computadoras, Supervisores, Procesamiento de Archivo Financiero, Jefe de Operadores de Comunicaciones,
Verificadores y Oficinistas de Créditos, Cantineros, Mozos y Mozas, Trabajadores de Cocina, Preparación de Comida, Guías, Ujieres, Graduadores y Sorteadores de Productos Agrícolas Excepto Explotación Forestal, Reparadores de Equipo de Procesamiento de Datos, Mecánicos de Molinos, Sastres, Zapateros, Empacadores a Mano, Operadores de Máquinas de Cementar y Encolar, Operadores de Máquinas de Elaboración Fotográfica, Supervisores, Operadores de Vehículos de Motor, Vendedores que utilizan Automóviles, Ingenieros de Operaciones, Operadores de Equipo de Estibadores, Operadores de Máquinas de Excavación y de Carga 


\section{Apéndice 2}

Tabla 1 - Ocupaciones con 100 por ciento de participación masculina, 1960

Administradores de Propiedades y

Bienes Raíces; Arquitectos; Ingenieros Mecánicos; Ingenieros Aeroespaciales; Ingenieros Petroleros; Ingenieros Químicos; Ingenieros Metalúrgicos y de Materiales; Ingenieros Nucleares; Ingenieros Industriales y ventas; Agrimensores y Científicos Cartográficos; Actuarios; Científicos Atmosféricos y Espaciales; Veterinarios; Especialistas en Relaciones Públicas; Archivistas y Curadores; Técnicos de Ingeniería Mecánica; Demostradores, Promotores y Modelos; Subastadores; Ocupaciones de Cortar Madera y Cortar y Transportar Madera; Pescadores; Cazadores y Tramperos; Reparadores de Enseres del Hogar y Herramientas de Fuerza Motriz; Mecánicos de Equipo Agrícola; Reparadores de Máquina de Oficina; Mecánicos de Molinos; Instaladores y Reparadores de Energía Eléctrica; Instaladores de Alfombras y Cortinas; Terminadores de Concreto y Terrazo; Empapeladores; Fabricantes de Herramientas y Troqueles; Trabajadores en Láminas de Metal; Fabricantes de Patrones y Modelos, Metal; Moldeadores Manuales, Excluyendo Joyeros; Fabricantes de Patrones, Trabajadores de Trazado y Cortadores; Fotograbadores y Litógrafos; Operadores de Máquinas de Extracción y Torceadores; Operadores de Hornos y Estufas, excluyendo Comida; Ayudantes, Rama de Construcción; Pilotos de Áviones y Navegadores; Directores de Tráfico Aéreo; Operadores de Equipo Periférico; Operadores de Máquinas de Cobros, Postero y Calculadoras; Oficiales de Justicia, Alguaciles y Otros Oficiales encargados de la Ejecución de la Ley; Podiatras, Técnicos de Ingeniería Mecánica; Operadores de Ferrocarril y Jefes de Patio; Ocupaciones de Operación de Locomotoras; Operadores de Frenos, Señales y Cambios de Vía del Tren 


\section{Tabla 2 - Ocupaciones con 100 por ciento de participación masculina,} 1980

Administradores de Servicios de Protección; Ingenieros Marinos y Arquitectos Navales; Ingenieros de Venta; Supervisores de Ocupaciones de Inspección y Prevención Fuegos; Porteros de Equipaje y Botones; Trabajadores forestales, excluyendo Transportar y Cortar Madera; Capitanes y Otros Oficiales, Barcos de Pesca; Mecánicos de Guaguas, Camiones y Motores Fijos; Reparadores de Motores Pequeños; Mecánicos de Aviación, excluyendo Motor; Aprendiz de Mecánica de Automóvil; Cerrajeros y Reparadores de Cajas de Seguridad; Supervisores: Carpinteros y Trabajos y Relacionados; Supervisores:

Albañiles, Mamposteros, Instaladores de Losetas; Supervisores: Pintores, Empapeladores, Empañetadores; Supervisores: Plomeros, Instaladores de Tubería y Máquinas de Vapor;
Aprendiz de Carpintero; Aprendiz de Electricista; Aprendiz de Plomero, Instalador de Tubería y Máquina de Vapor; Aprendiz de Albañil y Mampostero; Instaladores de Paredes de Yeso Seco; Trabajadores en Insolación; Operadores de Equipo de Pavimentación, Allanamiento y Apisonamiento; Constructores de Techos; Instaladores de Tubos de Metal; Supervisores, Ocupaciones de Extracción; Trabajadores con Explosivos; Trabajadores de Trazado; Trabajadores de Precisión en Madera; Operadores de Máquinas de Procesar Metal y Plástico, Misceláneos; Operadores de Máquinas de Carpintería; Ocupaciones de Operadores de Vehículos de Motor; Operadores de Equipo de Estibadores; Supervisores, Manipuladores, Limpiadores de Equipo y Obreros; Ayudantes, Agrimensor 